\title{
Development of the $(\mathrm{d}, \mathrm{n})$ proton-transfer reaction in inverse kinematics for structure studies*
}

\author{
K.L. Jones ${ }^{a}$, C. Thornsberry $a$, J. $\operatorname{Allen}^{b}$, A. Atencio $^{c}$, \\ D.W. Bardayan ${ }^{b}$, D. Blankstein ${ }^{b}$, S. Burcher $^{a}$, A.B. Carter ${ }^{a}$, \\ K.A. Chipps ${ }^{d}$, J.A. Cizewski ${ }^{c}$, I. Cox $^{a}$, Z. Elledge ${ }^{a}$, \\ M. Febbraro ${ }^{d}$, A. Fijalkowska ${ }^{c}$, R. Grzywacz ${ }^{a}$, M.R. Hall $^{b}$, \\ T.T. King ${ }^{a}$, A. Lepailleur ${ }^{c}$, M. Madurga ${ }^{a}$, S.T. Marley ${ }^{e}$, \\ P.D. O'Malley ${ }^{b}$, S.V. Paulauskas ${ }^{a}$, S.D. Pain ${ }^{d}$, W.A. Peters ${ }^{f}$,

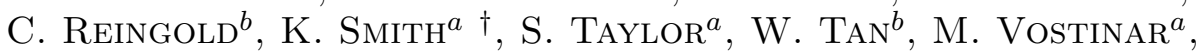 \\ D. WALTER ${ }^{c}$ \\ ${ }^{a}$ Department of Physics and Astronomy, University of Tennessee, Knoxville, \\ TN 37996, USA \\ ${ }^{b}$ Department of Physics, University of Notre Dame, Notre Dame, IN 46556, USA \\ ${ }^{c}$ Department of Physics and Astronomy, Rutgers University, New Brunswick, \\ NJ 08903, USA \\ ${ }^{d}$ Physics Division, Oak Ridge National Laboratory, Oak Ridge, TN 37831, USA \\ ${ }^{e}$ Department. of Physics Louisiana State University, USA \\ ${ }^{f}$ Joint Institute for Nuclear Physics and Applications, Oak Ridge, TN 37831, USA
}

Transfer reactions have provided exciting opportunities to study the structure of exotic nuclei and are often used to inform studies relating to nucleosynthesis and applications. In order to benefit from these reactions and their application to rare ion beams (RIBs) it is necessary to develop the tools and techniques to perform and analyze the data from reactions performed in inverse kinematics, that is with targets of light nuclei and heavier beams. We are continuing to expand the transfer reaction toolbox in preparation for the next generation of facilities, such as the Facility for Rare Ion Beams (FRIB), which is scheduled for completion in 2022. An important step in this process is to perform the $(\mathrm{d}, \mathrm{n})$ reaction in inverse kinematics, with analyses that include Q-value spectra and differential cross sections. In this way, proton-transfer reactions can be placed on the same level as the more commonly used neutron-transfer reactions, such as (d,p), $\left({ }^{9} \mathrm{Be},{ }^{8} \mathrm{Be}\right)$, and $\left({ }^{13} \mathrm{C},{ }^{12} \mathrm{C}\right)$. Here we present an overview of the techniques

\footnotetext{
* Presented at the XXXV Mazurian Lakes Conference on Physics, Piaski, Poland, September 3-9, 2017

${ }^{\dagger}$ Current address: Los Alamos National Laboratory, Los Alamos, NM 87545, USA
} 
used in $(\mathrm{d}, \mathrm{p})$ and $(\mathrm{d}, \mathrm{n})$, and some recent data from $(\mathrm{d}, \mathrm{n})$ reactions in inverse kinematics using stable beams of ${ }^{12} \mathrm{C}$ and ${ }^{16} \mathrm{O}$.

PACS numbers: 25.60.Je, 29.38.Gj, 21.10.Pc, 21.10.Jx, 25.45.Hi

\section{Introduction: Deuteron-induced transfer reactions}

Deuteron

Proton and neutron;

Tensor force brings together

fragile building block.

Transfer reactions can be used to elucidate the structure of nuclei through measurements of the Q-value of the reaction, and the intensity and shape of angular distributions of particles emerging from the reaction. When rare ion beams (RIBs) undergo transfer reactions on targets of light ions, commonly referred to as inverse kinematics measurements (see Fig. 1), the structure of exotic nuclei can be studied in detail. The first neutron transfer reactions performed in inverse kinematics used stable ${ }^{132,136}$ Xe beams at the Gesellschaft für Schwerionenforschung (GSI) 11. This pioneering experiment laid the groundwork for many neutron transfer studies performed with RIBs since the mid-1990s, in particular using the (d,p) single-neutron transfer reaction.

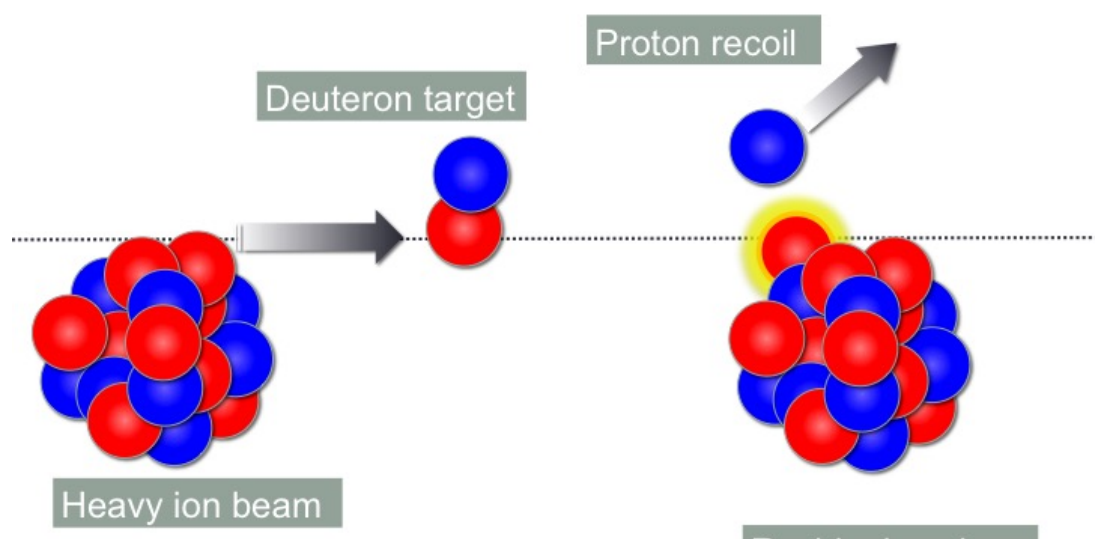

Residual nucleus

Fig. 1. Cartoon of a $(\mathrm{d}, \mathrm{p})$ reaction in inverse kinematics. A RIB impinges on a deuteron target resulting in a residual nucleus and a proton. Adapted from Ref. 2] 
The Q-value of a $(\mathrm{d}, \mathrm{p})$ reaction is extracted from the measured angle and energy of the proton. The conversion from the laboratory to the centerof-mass frame causes kinematic compression of the spectrum of states in the residual nucleus. However, in most cases the limiting factor in the resolution is the $\mathrm{CD}_{2}$ target thickness, and commonly targets of $100-200 \mu \mathrm{g} / \mathrm{cm}^{2}$ are used in order to resolve individual states, while still achieving statistical significance. Thicker targets can be used when states are identified from their $\gamma$ decays (see for example Refs. $[3]$ ), but in this case the statistical uncertainty is often limited by the efficiency of the $\gamma$-ray detector system.

The shapes of the angular distributions of protons emitted from $(\mathrm{d}, \mathrm{p})$ reactions can be indicative of the $\ell$ transfer of the reaction. The sensitivity of the angular distributions to the transferred $\ell$ is dependent on the beam energy, with very low or very high beam energies (compared to the energy of the Coulomb barrier) producing fairly flat angular distributions, regardless of $\ell$ transfer.

Another quantity of interest that can be extracted from transfer reactions is the spectroscopic factor, $S$, which is connected to the structure of the nucleus through the single-particle radial overlap function $u_{\ell s j}$ and the normalized wave function, $v_{\ell s j}$ :

$$
S_{\ell s j}=\left|A_{\ell s j}\right|^{2}
$$

where $\mathrm{A}_{\ell s j}$ is the spectroscopic amplitude, and:

$$
u_{\ell s j}(r)=A_{\ell s j} v_{\ell s j}(r) .
$$

However, as $S$ is extracted from a measured normalized differential crosssection and an angular distribution calculated from a reaction model:

$$
S_{\text {exp }}=\frac{d \sigma_{\text {exp }} / d \Omega}{d \sigma_{\text {calc }} / d \Omega}
$$

$S$ is not an observable of the experiment and is model dependent. The main sources of uncertainty in the calculations come from the optical (scattering) potentials and the bound state potential in the final state. Optical potentials can be extracted by fitting elastic scattering data. Different optical potentials can produce angular distributions with both different shapes and intensities. As for the bound state of the residual nucleus, this is typically modeled by a Woods-Saxon potential with the depth adjusted to the binding energy of the state, and the geometry defined by the radius and diffuseness. At beam energies close to the Coulomb barrier the magnitudes of the calculated differential cross sections can be very sensitive to the bound-state potential used. 


\section{The $(d, n)$ single proton transfer reaction}

In stark contrast to the recent growth in inverse kinematics $(d, p)$ measurements, there have been fewer developments made for the $(\mathrm{d}, \mathrm{n})$ singleproton transfer reaction, in particular where the nuclear structure is extracted from measuring the emitted neutrons (see for example [9 11]). Our collaboration has been developing techniques for performing the $(\mathrm{d}, \mathrm{n})$ proton transfer reaction with RIBs using the Versatile Array of Neutron Detectors at Low Energy (VANDLE) [12, 13. The energy and angle of protons emitted from $(\mathrm{d}, \mathrm{p})$ reactions can be measured with relative ease in silicon detectors. It is less straightforward to measure the energy of neutrons emerging from $(\mathrm{d}, \mathrm{n})$ reactions. VANDLE uses the time of flight of neutrons to find their kinetic energy. However, the plastic scintillator from which VANDLE is built is also sensitive to $\gamma$ rays, which can cause a limiting source of background in RIB experiments.

Our first attempt to measure states in ${ }^{8} \mathrm{~B}$ using the ${ }^{7} \mathrm{Be}(\mathrm{d}, \mathrm{n}){ }^{8} \mathrm{~B}$ reaction, at the TWINSOL facility at the University of Notre Dame [14], suffered from excessive levels of background in the neutron spectra. This was due in part to the configuration where the VANDLE bars were in the direct path of neutrons and $\gamma$ rays emitted from the RIB source in TWINSOL. A measurement made in the same experimental campaign utilizing the ${ }^{17} \mathrm{~F}(\mathrm{~d}, \mathrm{n})^{18} \mathrm{Ne}$ reaction produced results from deuterated liquid scintillator detectors 15 where pulse-shape discrimination (PSD) was used to separate neutrons from $\gamma$ rays. This largely eliminated the background issues. However, a limitation in this technique is the requirement that neutrons deposit energy above a threshold of about 100 keVee before PSD separation can be achieved. For comparison, in the analysis presented below the threshold for VANDLE was set at $30 \mathrm{keVee}$, allowing neutrons with energies down to $300 \mathrm{keV}$ to be measured. As the most interesting region of the angular distribution is at backward angles in the laboratory frame where the neutrons have the lowest energies, this threshold can severely limit the PSD technique. Liquid neutron detectors can still be used in as TOF detectors below neutron energies of about $1 \mathrm{MeV}$.

Ideally, these reactions would be performed in a configuration where the detectors are shielded from both the neutrons and the $\gamma$ rays emanating from the production target, the beam dump would not be directly viewed by the detectors, and the room would have low levels of background radiation. In most cases it is necessary to tag on the reaction of interest, usually by requiring an increase in charge of the recoil by one unit compared to the beam species. As the recoil cone is small and forward focussed this requires using 
a detector system that has $Z$-discrimination and can take the full beam rate. Options include phoswich (phosphor sandwich detectors), ionization chambers, magnetic recoil separators, and time-of-flight setups. The choice of recoil detector depends on the specifics of the experiment, namely the beam rate and the $\Delta Z / Z$ (i.e. $1 / Z$ ), the latter of which is straightforward for light nuclei, but becomes increasingly stringent for heavier elements.

Since these first attempts at ${ }^{7} \mathrm{Be}(\mathrm{d}, \mathrm{n}){ }^{8} \mathrm{~B}$ and ${ }^{17} \mathrm{~F}(\mathrm{~d}, \mathrm{n}){ }^{18} \mathrm{Ne}$ measurements, there have been significant upgrades to TWINSOL, with more shielding between the source and the experimental area [16]. At the same time, the production efficiency has been increased by reducing the thickness of the gas cell windows, and the ion optics have been improved to provide a smaller beam spot. Additionally, recoil identification detectors will aid in channel selection to reduce background in future VANDLE measurements with RIBs.

\section{Inverse Kinematics $(d, n)$ reactions with stable beams}

A stable beam experiment was run at the Nuclear Structure Laboratory (NSL) at the University of Notre Dame in preparation for future TWINSOL experiments and to investigate the response of VANDLE to inverse kinematics $(\mathrm{d}, \mathrm{n})$ reactions where signal-to-noise levels are more optimal than with RIBs and the level of statistics is not limiting. A total of 21 VANDLE detectors were mounted, at a distance of $0.5 \mathrm{~m}$ from a deuterated polyethylene $\left(\mathrm{CD}_{2}\right)$ target, at laboratory angles from $65^{\circ}$ to $170^{\circ}$. The stop signal for the time of flight (ToF) was provided by the delayed RF signal from the sweeper/buncher after the FN tandem. The bunches came at $100 \mathrm{~ns}$ intervals. The beams used were ${ }^{12} \mathrm{C}$, at 8 energy steps between 18.5 and $41.7 \mathrm{MeV}$, and ${ }^{16} \mathrm{O}$ at $64 \mathrm{MeV}$. A $413 \mu \mathrm{g} / \mathrm{cm}^{2} \mathrm{CD}_{2}$ target was used for both beams, a second, thicker $711 \mu \mathrm{g} / \mathrm{cm}^{2} \mathrm{CD}_{2}$ target was used for the ${ }^{12} \mathrm{C}$ beam. In total, approximately 2 hours of beam were used for the 8 energy steps with the ${ }^{12} \mathrm{C}$ beam, and an hour for the ${ }^{16} \mathrm{O}$. The beam intensities were 4 $5 \mathrm{enA}$ in each case, which represents about $1-5 \times 10^{9} \mathrm{pps}$.

Fig. 2 shows the type of spectra seen online from just a few minutes of beam, ${ }^{16} \mathrm{O}$ in this case. Each detector covered approximately $5^{\circ}$ in the laboratory frame, with the most backward angles, from $170^{\circ}$, at the bottom of the figure. The two vertical lines, where all detectors fired at the same ToF regardless of angle, are the $\gamma$ flashes from the target and the beam stop. The target $\gamma$ flash was used as a timing reference for measuring the kinetic energy of the neutrons. The two loci relating to the proton-transfer to the 


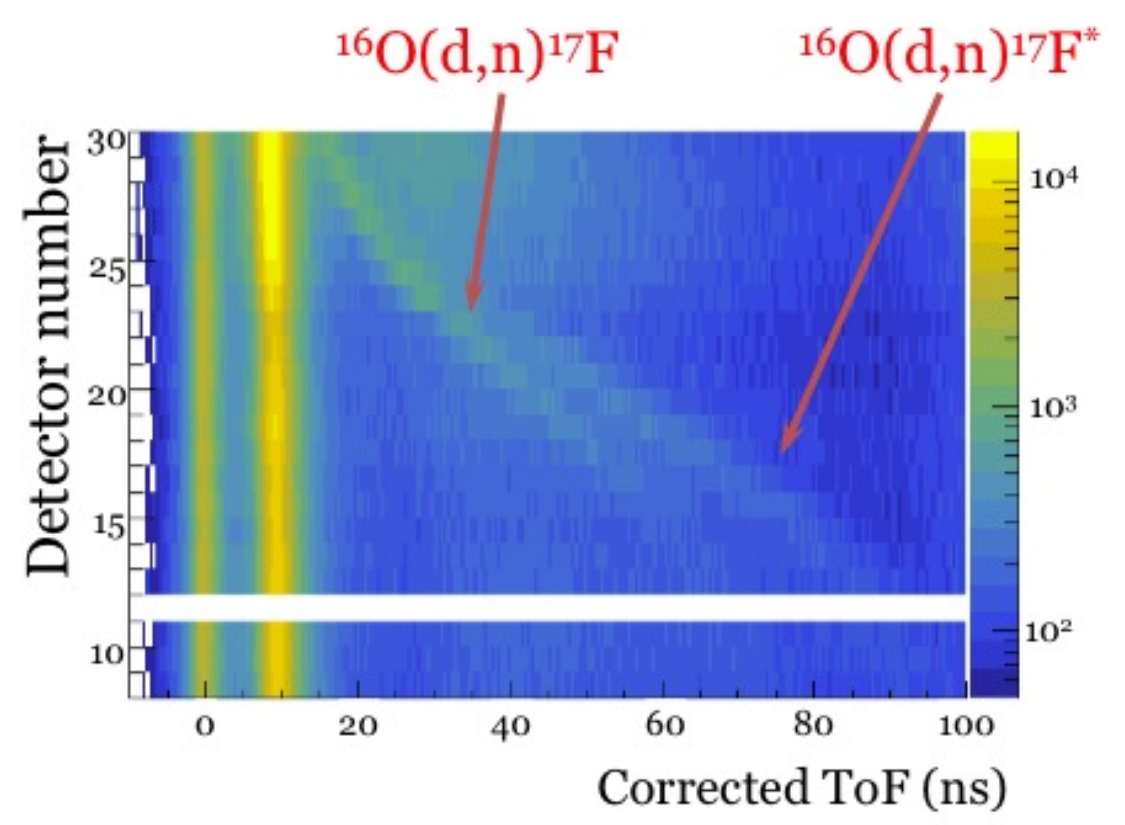

Fig. 2. Detector number versus corrected time of flight for neutrons following the ${ }^{16} \mathrm{O}(\mathrm{d}, \mathrm{n}){ }^{17} \mathrm{~F}$ reaction, in inverse kinematics, at $\mathrm{E}_{{ }^{16} \mathrm{O}}=64 \mathrm{MeV}$. The polar angle in the laboratory frame increases with decreasing detector number. The detectors covered a range from $65^{\circ}$ to $170^{\circ}$ in the laboratory frame. The two vertical lines at $0 \mathrm{~ns}$ and $10 \mathrm{~ns}$ are from the flashes of $\gamma$ rays emitted as the beam struck the target and the beam stop, respectively.

ground and first excited states in ${ }^{17} \mathrm{~F}$ traverse the plot from the top left, close to the beam stop $\gamma$ flash toward the bottom right, where the intensity fades to around that of the background.

Fig. 3 shows the same information, but for the ${ }^{12} \mathrm{C}(\mathrm{d}, \mathrm{n}){ }^{13} \mathrm{~N}$ reaction, after the conversion of the neutron ToF into energy and detector number into the laboratory angle. The ground state population is seen as a locus sweeping across from the top-center of the plot toward the lower right. As in inverse kinematics $(\mathrm{d}, \mathrm{p})$ measurements, the emergent particle has higher energies at forward angles and low energies at backward angles.

The data for each beam at each beam energy were subdivided by polar angle of the neutron in the center-of-mass frame. The intensity of each state was found by fitting a gaussian curve on a linear background, as shown in Fig. 4. These fits were made on the neutron ToF spectra as the resolution is linear, as apposed to neutron energy spectra where the resolution goes 


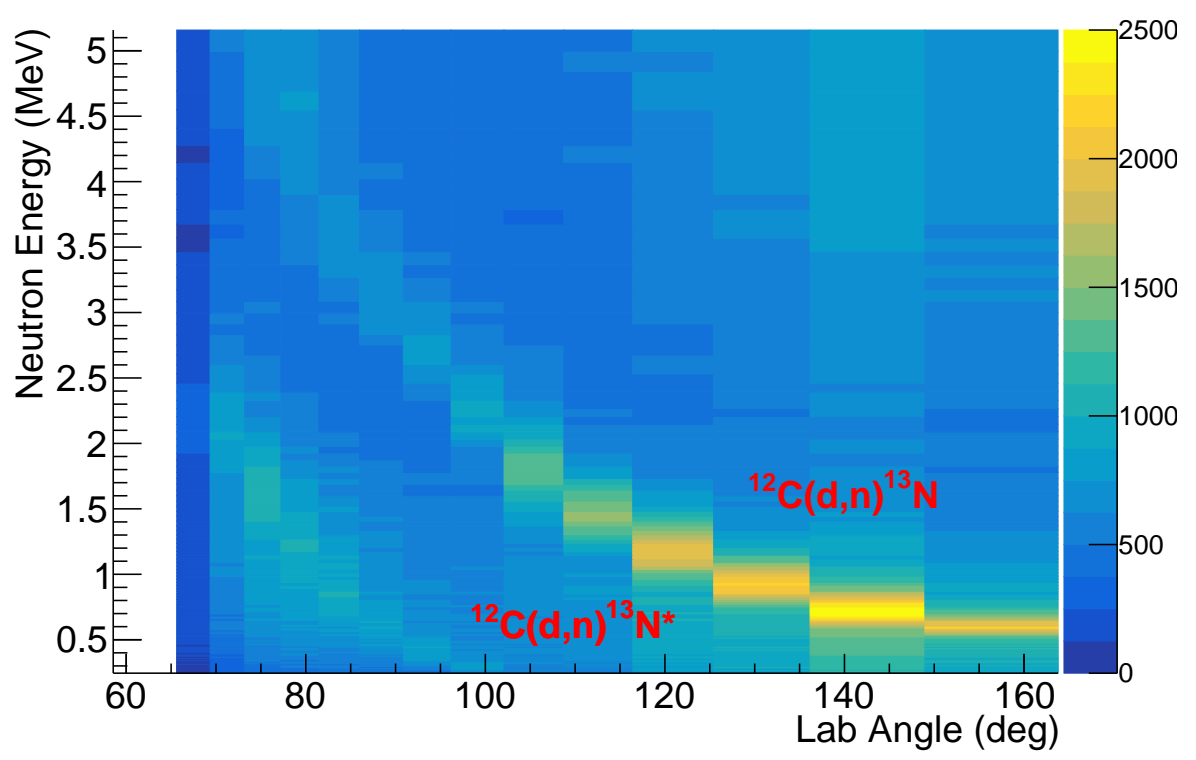

Fig. 3. Angle versus energy plot for neutrons following the ${ }^{12} \mathrm{C}(\mathrm{d}, \mathrm{n}){ }^{13} \mathrm{~N}$ reaction, in inverse kinematics, at $\mathrm{E}_{12} \mathrm{C}=41.7 \mathrm{MeV}$. The loci show the population of the ground state and an excited state at $2.365 \mathrm{MeV}$. Smoothing related to the width of each detector was applied in the calculation of the polar angle.

as $\mathrm{ToF}^{2}$. These intensities can then be plotted versus center-of-mass angle to produce angular distributions. A normalization was performed using the known target thickness and the beam intensity, monitored by a current integrator connected to an electrically insulated brass beam stop. The differential cross sections are currently being finalized and are beyond the scope of this contribution. Those that have been analyzed are of sufficient quality to make meaningful comparisons with previous data and state-ofthe-art theory.

\section{Outlook}

The $(\mathrm{d}, \mathrm{p})$ reaction has been used extensively in inverse kinematics since the mid-1990's, thus allowing transfer reaction techniques to be performed on beams of exotic nuclei. This has proven to be a powerful method for extracting spectroscopic information relating to single-neutron states away from stability. In order to study single-proton states in an analogous way it is necessary to develop techniques relating to the $(\mathrm{d}, \mathrm{n})$ single-proton transfer reaction. Our collaboration performed the $(\mathrm{d}, \mathrm{n})$ reaction on beams of 


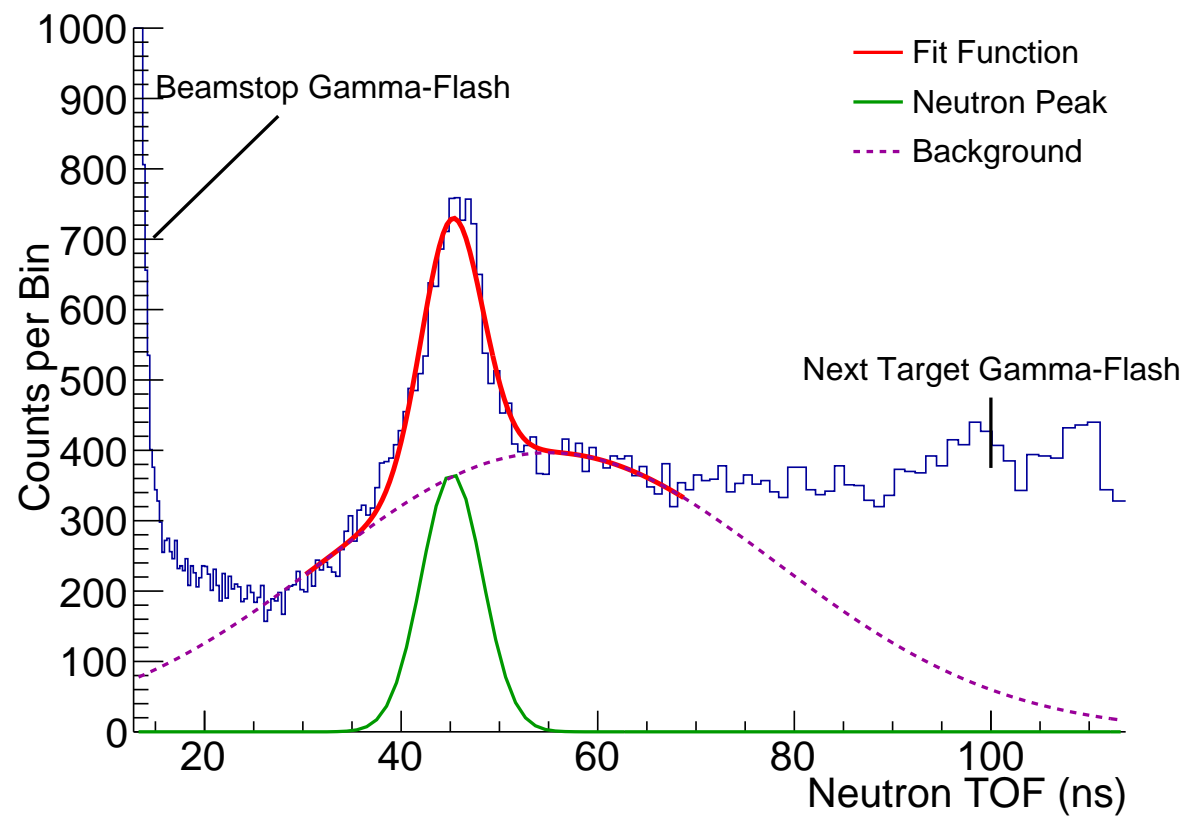

Fig. 4. Neutron time-of-flight spectrum for the ${ }^{12} \mathrm{C}(\mathrm{d}, \mathrm{n})^{13} \mathrm{~N}$ reaction at $\theta_{C M}=12^{\circ}$ $\left(\theta_{l a b}=144^{\circ}\right)$, in inverse kinematics, with a beam energy of $\mathrm{E}_{12} \mathrm{C}=41.7 \mathrm{MeV}$. The gaussian fit to the peak for population of the ground state, the background, and the sum fit function are shown in green, magenta, and red respectively. The timing of the $\gamma$ flash from the next beam pulse is shown.

stable ${ }^{12} \mathrm{C}$ and ${ }^{16} \mathrm{O}$ with the neutron ToF array VANDLE. High quality data have been extracted from these measurements and normalized differential cross-sections are currently being finalized.

Channel selection is important to VANDLE measurements with RIBs, where backgrounds from both neutrons and $\gamma$ rays are often limiting. Currently we are developing a compact ionization chamber with an internal scintillator to provide a tag on the $Z$ of the recoil. The scintillator will be instrumented with silicon photomultipliers. The results presented here from stable beam measurements are encouraging and provide motivation to develop recoil particle identification detectors such that the $(\mathrm{d}, \mathrm{n})$ reaction, measured with ToF detector arrays, can be used as a spectroscopic tool with RIBs. 


\section{Acknowledgments}

This research was supported in part by the U.S. Department of Energy, Office of Science, Office of Nuclear Physics under Contract No. DEFG02-96ER40963 (UT), DE-AC05-00OR22725 (ORNL), by the National Science Foundation under Contract No. PHY1713857 (ND), PHY0354870 and PHY1404218 (Rutgers). This research was sponsored in part by the National Nuclear Security Administration under the Stewardship Science Academic Alliance program through DOE Cooperative Agreement No. dena0002132 (Rutgers and UTK). STM would like to acknowledge funding from the Louisiana State University Department of Physics and Astronomy.

\section{REFERENCES}

[1] G. Kraus et al., Z. Phys. A340, 339 (1991).

[2] K.L. Jones, Phys. Scr. T152, 014020 (2013).

[3] M. Labiche et al., Nucl. Instr. and Meth. in Phys. Res. A614, 439 (2010).

[4] J. Simpson et al., Acta Physica Hungarica 11, 159 (2000).

[5] V. Bildstein et al., Prog. in Part. and Nucl. Phys. 59, 386 (2007).

[6] J. Eberth et al., Progress in Particle and Nuclear Physics 46, 389 (2001).

[7] C. A. Diget et al., Journal of Instrumentation 6, P02005 (2011).

[8] C. Svensson et al., J. Phys. G 31, S1663 (2005).

[9] W.A. Peters et al., Bull. Am. Phys. Soc. 58, 29 (2013).

[10] M. Febbraro et al., Phys. Rev. C 96, 024613 (2017).

[11] L. Baby et al., Nucl.Instr. and Meth. in Phys. Res. A 877, 34 (2018).

[12] S.V. Paulauskas et al., Nucl. Instr. and Meth. in Phys. Res. A 737, 22 (2014).

[13] W.A. Peters et al., Nucl. Instr. and Meth. in Phys. Res. A836, 122 (2016).

[14] F.D. Becchetti et al., Nucl. Instr. and Meth. in Phys. Res. B 376, 397 (2016).

[15] M. Febbraro et al., EPJ Web of Conferences 66, 03026 (2014).

[16] P.D. O'Malley et al., Nucl. Instr. and Meth. in Phys. Res. B 376, 417 (2016). 